\title{
S'appuyer sur le passé pour construire l'avenir : l'exemple du projet nationaliste écossais dans le domaine des énergies renouvelables offshore
}

Building the future on the past: the example of the Scottish nationalist project in the field of offshore renewable energies

\section{Sylvain Roche}

\section{(2) OpenEdition Journals}

Édition électronique

URL : http://journals.openedition.org/artefact/4086

DOI : 10.4000/artefact.4086

ISSN : 2606-9245

Éditeur :

Association Artefact. Techniques histoire et sciences humaines, Presses universitaires du Midi

Édition imprimée

Date de publication : 15 octobre 2019

Pagination : $143-174$

ISBN : 978-2-8107-0650-1

ISSN : 2273-0753

\section{Référence électronique}

Sylvain Roche, «S'appuyer sur le passé pour construire l'avenir : l'exemple du projet nationaliste écossais dans le domaine des énergies renouvelables offshore ", Artefact [En ligne], 10 | 2019, mis en ligne le 06 août 2020, consulté le 27 novembre 2020. URL : http://journals.openedition.org/artefact/ 4086 ; DOI : https://doi.org/10.4000/artefact.4086

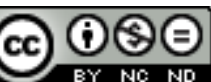

Artefact, Techniques, histoire et sciences humaines est mise à disposition selon les termes de la Licence Creative Commons Attribution - Pas d'Utilisation Commerciale - Pas de Modification 4.0 International. 


\section{S’appuyer sur le passé pour construire l'avenir : l'exemple du projet nationaliste écossais dans le domaine des énergies renouvelables offshore}

\section{Sylvain Roche}

\section{Résumé}

Outre sa souveraineté politique, l'Écosse cherche à conquérir son indépendance énergétique. Au cœur d'un projet qui se veut résolument écologique, les énergies renouvelables offshore sont devenues progressivement un synonyme de rayonnement politique, scientifique et médiatique, pour une nation qui a toujours placé les avancées technologiques au cœur de son histoire. Les énergies renouvelables s'intègrent en effet dans la mythologie des nationalistes écossais : un pays riche de ressources inépuisables, comme le vent, les vagues et les courants de marées. Après le pétrole, les énergies renouvelables représenteraient le deuxième conte merveilleux de l'Écosse. En quoi le développement des énergies renouvelables offshore peut-il se définir comme un projet émanant d'un discours d'affirmation nationale?

\section{Mots-clés}

Écosse, énergies marines renouvelables, innovation technologique, politique énergétique, nationalisme des ressources naturelles, techno-nationalisme, technopolitique

95 Sylvain Roche, « S'appuyer sur le passé pour construire l'avenir : l'exemple du projet nationaliste écossais dans le domaine des énergies renouvelables offshore », Artefact, 10, 2019, p. 143-174. 


\section{Building the future on the past: the example of the Scottish nationalist project in the field of offshore renewable energies}

\section{Abstract}

In addition to its political sovereignty, Scotland seeks to conquer its energy independence. In a project that is resolutely environmentally friendly, offshore renewable energies have gradually become synonymous with political, scientific and media influence, for a nation that has always placed the new technologies at the heart of its history. Renewable energies are part of the Scottish nationalist mythology: a country rich in inexhaustible resources such as wind, waves, and tidal currents. After oil extraction and the success story of electronics, renewables would be Scotland's second wonderful tale. To what extent is the development of offshore renewable energy production capacity in Scotland part of a project (political discourse) of national affirmation?

\section{Keywords}

Energy policy, marine renewable energy, resource nationalism, Scotland, technological innovation, technological nationalism, technopolitics

\section{Note de l'auteur}

Nous considérons comme énergies renouvelables offshore : l'éolien en mer (offshore wind), l'énergie des vagues (wave energy) et l'énergie des marées et courants de marée (tidal energy). 


\section{Introduction}

Depuis bientôt vingt ans, l'Écosse mène une politique énergétique ambitieuse pour décarboner son économie. Grâce à une politique d'installation d'infrastructures qui utilisent la force du vent et de l'eau, l'Écosse a produit $68,1 \%$ de son énergie électrique à partir de sources renouvelables ${ }^{1}$. Les énergies renouvelables ont connu un fort développement en Écosse ces dernières années, passant de $8 \%$ de la consommation finale d'énergie de ce territoire en 2009 à presque $18 \%$ en $2015^{2}$. Cette montée en puissance est essentiellement due au développement rapide et appuyé de l'éolien terrestre (307,8 MW installés en 2003 à $7544 \mathrm{MW}$ en 20173). Publiée le 20 décembre 2017, la dernière feuille de route énergétique écossaise vise à porter à $50 \%$ la part des énergies renouvelables dans la consommation finale d'énergie d'ici 2030 (incluant l'énergie thermique, l'énergie mécanique et l'énergie électrique). L'Écosse dispose de ressources énergétiques naturelles abondantes et d'une " tradition " de pionnière dans le secteur de l'industrie de l'énergie que de nombreux acteurs ont participé à construire et à valoriser. Elle a toujours compté sur ses richesses naturelles pour viser l'autonomie énergétique, qu'il s'agisse de ses ressources environnementales ou intellectuelles.

Pilotée par le Parti national écossais (Scottish National Party) depuis sa victoire aux élections parlementaires de 2007, la question énergétique reste très sensible en Écosse, et à ce titre, les énergies renouvelables ont largement couvert les débats lors de la campagne électorale précédant le référendum de 2014. Les énergies renouvelables offshore conjuguent à elles seules les grandes transformations idéologiques souhaitées par les nationalistes : une Écosse plus soucieuse de l'environnement, autonome d'un point de vue énergétique, dynamique sur le secteur des technologies de pointe et plus européenne. L'espoir suscité par les énergies offshore n'est donc pas étranger à la vive poussée de nationalisme constatée en Écosse dans les années 2000. À ce titre, l'Écosse fournit un cadre idéal à l'intérieur duquel poursuivre la technopolitique de l'identité nationale et de l'objectif du rayonnement, comme en témoignent les titres élogieux des journaux

1. " "Record” year for renewable electricity generation ", BBC News, 29 mars 2018.

2. The Scottish Government, 2017.

3. Digest of UK Energy Statistic 2018, Regional Statistics 2003-2017: Installed Capacity https:// www.gov.uk/government/statistics/regional-renewable-statistics Consulté le 26 avril 2019. 
français : «L'Écosse : poisson pilote des énergies marines ${ }^{4}$ "; « L'Écosse à la pointe des énergies marines " ; "L'Écosse bascule du pétrole vers les énergies marines ${ }^{6}$ "; " L'Écosse, la future Arabie saoudite écolo d'Europe ${ }^{7}$ ? " ; "L'Écosse se rêve en futur grand des énergies renouvelables ${ }^{8}$ "; "L'Écosse se veut championne de l'électricité propre " ${ }^{9}$. Depuis les études proposées par l'anthropologie des sciences et techniques ${ }^{10}$ et celles plus récentes en sociologie politique des techniques ${ }^{11}$, chacun reconnait que les " capacités de la technologie ne sont pas neutres ${ }^{12}{ }^{\prime}$. L'histoire montre en effet que l'innovation technologique n'est neutre ni politiquement ni socialement. Les projets technologiques ont des propriétés politiques qui leur sont propres et répondent à des objectifs politiques précis. L'historienne Gabrielle Hecht utilise le concept de technopolitique pour définir " les pratiques stratégiques qui consistent à concevoir ou à utiliser la technique afin de mettre en place des objectifs politiques, de leur donner forme et de les réaliser ${ }^{13}$ ". Les caractéristiques de la technopolitique dépendent néanmoins de l'environnement général dans lequel elle s'inscrit. Marco Adria parle de technological nationalism pour définir les liens entre les technologies et les projets politiques nationalistes : "Technological nationalism involves the explicit use of technology as the subject of a nationalist project's strategy [...] It combines the idea of technological progress with the sentiments and goals of nationalism ${ }^{14}$. " Pour l'historien David Edgerton : "Le techno-nationalisme suppose que l'unité de base pour l'étude de la technologie est la nation : les nations sont les unités qui inventent, qui ont des budgets de recherche et développement, des cultures novatrices, qui diffusent et utilisent les techniques ${ }^{15}$. " Ross Bond et al., dans leur article National Identity and Economic Development: Reiteration, Recapture, Reinterpretation and Repudiation (2003), montrent ainsi comment le nationalisme est utilisé

\footnotetext{
4. Libération, 21 octobre 2013.

5. BFM, 6 octobre 2014.

6. La Tribune, 8 mars 2012

7. Slate.fr, 13 septembre 2014.

8. Les Echos, 17 février 2010.

9. Le Monde, 5 décembre 2007.

10. Akrich, 1987 ; Latour, 1992.

11. Barthe, 2009; Hecht, 2014.

12. Piatti, 2001, p. 32.

13. Hecht, 2014, p. 24.

14. Adria, 2010, p. 45.

15. Edgerton, 2013, p. 149.
} 
pour porter des projets d'innovation et, plus spécifiquement, comment les symboles et les histoires nationales sont mobilisés à des fins politiques. La technopolitique s'articule en effet autour de valeurs communes telles que l'indépendance nationale et le prestige technologique.

En quoi le développement des énergies renouvelables offshore peut-il se définir comme un projet émanant d'un discours d'affirmation national ? Pour répondre à cette question, ce papier se décomposera en quatre parties. La première définira la technopolitique des énergies renouvelables offshore comme le couplage d'un nationalisme des ressources naturelles et d'un techno-nationalisme. Dans une seconde partie, nous verrons que ce nationalisme énergétique écossais s'inscrit dans un processus de modernisation idéologique à travers le projet de transition écologique. Dans une troisième partie, nous montrerons que ce nationalisme énergétique s'inscrit dans un modèle politico-économique endogène qui se veut de nature libéral et européen. Enfin, nous soulignerons dans une quatrième partie que ce nationalisme énergétique s'inscrit dans une stratégie de rayonnement par l'innovation technologique.

Cet article se base sur des sources diverses : travaux scientifiques, articles d'opinion, discours des responsables politiques, points de vue des éditorialistes, entretiens avec des acteurs, qu'il convient de critiquer et de confronter. Les médias ne sont pas toujours des outils de communication neutres ${ }^{16}$ mais ils nous permettent de mieux comprendre la construction des discours et des représentations collectives des technologies énergies marines. À ce titre, nous verrons que la presse reste un canal important où s'exprime la technopolitique écossaise des énergies marines, participant à estomper la distinction entre journalisme et marketing territorial.

\section{Faire de ses richesses naturelles un nationalisme énergétique}

L'histoire des liens entre ressources naturelles et nationalisme est à inscrire dans la longue durée. Depuis des siècles, sociétés et États ont utilisé certaines ressources naturelles afin de promouvoir leurs intérêts et de poursuivre leurs objectifs politiques ${ }^{17}$. L'histoire du capitalisme moderne serait

16. Joris, 2012.

17. Goetschel et Péclard, 2006. 
ainsi marquée par une suite continue de revendications plus ou moins violentes pour la maîtrise de la terre et des ressources naturelles ${ }^{18}$. Le principe de souveraineté sur les ressources naturelles a été introduit dans les débats des Nations Unies à la suite de la demande des pays colonisés et des pays en développement de pouvoir bénéficier de l'exploitation de leurs ressources naturelles ${ }^{19}$. Un long processus tenu au sein de l'ONU a fini par consacrer le principe de la souveraineté permanente sur les ressources naturelles. C'est ainsi que par sa célèbre résolution 1803 (XVII) du 14 décembre 1962, l'Assemblée générale a solennellement proclamé le droit de tout État de disposer librement de ses richesses et de ses ressources naturelles. Aujourd'hui, chaque État dispose, en droit, d'une souveraineté pleine et entière sur ses richesses et ressources naturelles. La Déclaration de Rio sur l'environnement et le développement de juin 1992 insiste sur le principe de souveraineté sur les ressources naturelles (principe 2) dans le développement des politiques de développement durable : "Conformément à la Charte des Nations Unies et aux principes du droit international, les États ont le droit souverain d'exploiter leurs propres ressources selon leur politique d'environnement et de développement. » Dans son article Nationalism and 148 Environmentalism, Paul Hamilton insiste sur la dimension nationaliste que portent intrinsèquement les politiques environnementales :

Nationalism and environmentalism are most closely joined in two ways. First, nationalists often use biogenetic metaphor to underpin their political demands [...] Second, nationalists will appeal to concerns about natural resources and link them to the overall health and future of the nation ${ }^{20}$.

L'environnement compte en effet parmi les institutions qui participent à la construction des identités nationales à l'époque moderne ${ }^{21}$. Il existerait un «nationalisme environnemental » dont le propre est d'établir un lien entre l'identité territoriale et l'environnement dans lequel il se déploie. En citant Nicola McEwen et Elizabeth Bomberg (2014):

By adopting the role of climate pioneer, a sub-state government can reinforce its territory's distinctiveness, assert the political importance of

18. Luna et Mignemi, 2017.

19. Rosenberg, 1983.

20. Hamilton, 2008, p. 877.

21. Forchtner et Kølvraa, 2015. 
the sub-state level, and use the economic opportunities offered by low carbon innovation and renewables as justification for the pursuit of greater political autonomy [...] As such, these forums not only offer opportunities to engage in multilateral decision-making and networking, but can contribute to strengthening territorial identity and nation-building.

Depuis près d'un siècle, l'énergie est assurément l'un des domaines où le nationalisme des ressources naturelles s'est manifesté avec le plus de vigueur et de constance ${ }^{22}$. La politique énergétique est un pouvoir hautement régalien et l'énergie reste un fort marqueur identitaire. Un État est légitime s'il maîtrise sa politique énergétique en étant garant de sa sécurité et de ses choix stratégiques. Claude Bellavance (2003) explique ainsi comment le développement de l'énergie hydraulique a été perçu au Québec comme un moyen d'émancipation nationale pour la majorité d'expression française. Des études historiques complémentaires ont montré comment le binôme ressources naturelles et innovations technologiques a participé à la diffusion d'un discours nationaliste québécois, permettant à l'État d'affirmer sa souveraineté et à des entreprises (Hydro-Québec) de croître $^{23}$. Ces travaux mettent d'ailleurs en exergue l'association entre la modernité technologique et l'émancipation politique, notamment portée par le Parti Québécois (PQ) dans les années 1970, l'équivalent du SNP écossais. Gabrielle Hecht (2014) nous montre quant à elle comment la technologie nucléaire a été construite après la Deuxième Guerre mondiale comme une composante fondamentale de l'identité nationale française. Ce nationalisme énergétique identitaire serait aujourd'hui un phénomène de plus en plus répandu depuis le tournant de l'industrie de l'énergie au tournant des années $2000^{24}$. Dans leur Livre blanc de la transition nationale de la Catalogne de 2014, les responsables politiques nationalistes ne manquèrent pas d'insister sur la mise en valeur des ressources hydrauliques de la communauté autonome pour viser l'indépendance nationale (rapport $\left.\mathrm{n}^{\circ} 9\right)^{25}$. Le discours nationaliste écossais s'inscrit dans ce lien complexe

22. Debeir et al., 2013.

23. Perron, 2006 ; Savard, 2009.

24. Hache, 2016 ; Bouzarovski et Bassin, 2011.

25. Du fait du parallélisme entre les processus indépendantistes catalan et écossais, il est légitime de s'interroger sur l'influence respective qu'ils ont pu exercer l'un sur l'autre sur ces questions d'énergie. 
entre territoire et puissance au travers de la valorisation de ressources naturelles énergétiques. En citant Mark Bailoni (2014) :

Les enjeux énergétiques exacerbent également certaines tensions et rivalités entre les territoires. Ils révèlent ainsi des clivages identitaires, sociaux et politiques spécifiques au Royaume-Uni, État-multi-nations [...] La question de l'énergie est donc au coeur des relations compliquées entre les nations composant le Royaume-Uni.

Là est une caractéristique forte du paysage énergétique britannique : pétrole, vent, marées ou vagues, la grande majorité de ces ressources énergétiques ont comme terrain de jeux les eaux écossaises ${ }^{26}$. Le nationalisme énergétique écossais a pris une dimension grandissante après la découverte dans les années 1970 des champs pétroliers de la mer du Nord. Pour les nationalistes, l'appartenance au Royaume-Uni empêche l'Écosse d'exploiter ses ressources naturelles abondantes en fonction de ses propres intérêts. En citant Paul Collier :

Dans de nombreux cas, les ressources naturelles sont situées dans des régions dont un groupe politique - bien que souvent marginal - revendique déjà l'autonomie. La présence de ressources naturelles permet à de tels groupes d'ajouter un argument économique crédible à ce qui n'est sinon certainement en grande partie qu'un appel « romanesque $»^{27}$.

L'augmentation (sans violence) du nationalisme écossais entre les élections de 1970 et 1974 illustre bien cette transformation. Le discours indépendantiste vise à convaincre l'opinion publique que les ressources naturelles de l'Écosse apporteront davantage de prospérité à ses habitants en cas d'indépendance. Lancée pour la première fois en 1974 par le SNP, l'idée que l'Écosse " possède " jusqu’à $90 \%$ des réserves pétrolières britanniques de la mer du Nord a toujours été l'une des principales revendications des nationalistes ${ }^{28}$. Pour le SNP, si la rente pétrolière tombait dans l'escarcelle d'Edimbourg plutôt que de Londres, la prospérité de l'Écosse serait dès

26. L'Écosse possède $25 \%$ du potentiel éolien offshore et marémoteur de l'UE et $10 \%$ de son potentiel en matière d'énergie des vagues : https://www.sdi.co.uk/business-in-scotland/key-sectors/ renewables, consulté le 26 avril 2019.

27. Collier, 2004, p. 201.

28. «Who has a right to claim North Sea oil? », BBC Scotland news, 16 avril 2013. 
lors assurée ${ }^{29}$. Ce discours vise à légitimer le rôle d'un État indépendant, celui d'un bon gestionnaire des rentes pétrolières faisant l'interface entre les multinationales chargées de l'exploitation des ressources naturelles et la population, dans une optique de redistribution partagée de la richesse engendrée, à l'image du modèle norvégien (Fig. 1). It's Scotland's oil reste l'un des slogans politiques les plus populaires dans le pays, un carburant toujours efficace du nationalisme écossais comme en témoignaient les débats lors de la campagne du référendum $2014^{30}$.

\section{Moderniser le nationalisme énergétique par le projet de transition écologique}

La crise économique et financière de 2008 fait entrer les énergies marines dans une phase dite de slope of enlightenment (pente de l'illumination). D'une part, la crise a accentué les liens entre les questions économiques et sociales en Écosse, participant à consolider le discours nationaliste du $\mathrm{SNP}^{31}$. D'autre part, elle a encouragé à développer de nouveaux viviers de croissance en faveur de la construction d'une nouvelle économie verte écossaise ${ }^{32}$. Trouver des voies concrètes de la réindustrialisation devient une priorité, et à ce titre, les énergies marines semblent offrir à l'Écosse des possibilités pour se construire un first advantage à l'échelle internationale, et notamment par rapport au voisin anglais ${ }^{33}$. Pour Gerry Hassan et Simon Barrow (2017):

A dominant narrative for the SNP administration was the benchmarking of Scotland's performance against the UK as a whole [...] Important to this was talking positively about the re-industrialisation of Scotland, building on its potential in renewable energy.

En 2010, le SNP publiait une stratégie bas carbone ambitieuse encourageant le développement des énergies renouvelables, dont les énergies offshore ${ }^{34}$. En 2013, l'Écosse se fixait l'objectif de produire à partir d'unités

29. Mechlin, 2015.

30. McEwen, 2014.

31. Camp-Pietrain, 2016.

32. The Scottish Government, 2010.

33. The Scottish Government, Reindustrialising Scotland for the 21 $1^{\text {th }}$ Century: a sustainable Industrial Strategy for a Modern Independant Nation, Edinburgh, 2014.

34. The Scottish Government, 2010. 
de production renouvelables "l'équivalent de $100 \%$ de ses besoins électriques » d'ici $2020^{35}$. Un arsenal de dispositifs publics visant à promouvoir les énergies renouvelables en mer (mécanismes financiers incitatifs, aides à la $\mathrm{R} \& \mathrm{D}$, etc.) était mis en place pour tenter d'exploiter les ressources naturelles nationales à leur plein potentiel. Cette politique doit aboutir à la construction d'un modèle énergétique écossais d'ici 2050 tourné vers les énergies renouvelables ${ }^{36}$, à l'image de ce que fut le modèle français tourné vers le nucléaire ou le modèle québécois avec l'hydraulique.

Les discours nationalistes s'appuient généralement sur des promesses de prospérité économique ${ }^{37}$. Ces énergies émergentes à fort potentiel marchand vont offrir au SNP l'occasion de porter des " discours promettants " et de persuasion auprès de la société ${ }^{38}$. Les perspectives solides de création d'un secteur industriel compétitif à l'exemple de l'éolien danois et allemand $^{39}$ ou du solaire japonais ${ }^{40}$ encouragent les acteurs publics à faire la promotion de ces énergies. Pour le SNP, les énergies renouvelables pourraient générer un revenu de 14 milliards de livres à l'horizon 2050 et contribuer à la création de dizaines de milliers d'emplois (près de 30000 pour le seul éolien offshore ${ }^{41}$ ). L'objectif affiché est d'atteindre 1,6 GW d'énergie des vagues et marémotrice déployée d'ici $2020^{42}$, soulignant à quel point l'enthousiasme et les espoirs sont alors énormes ${ }^{43}$. Ces énergies émergentes à fort potentiellement marchant vont de plus bénéficier d'un consensus politique pour leur développement. En citant Cowell et al. :

The expansion of renewable energy has attracted support in Scotland across the main three political parties—SNP, Labour and the Liberal Democrats - from the first days of devolved government [...] This temporal continuity in political resources created the time for governing

35. The Scottish Government, 2013a.

36. The Scottish Government, 2017.

37. Keating, 2001.

38. Voir à ce titre l'ouvrage de Marc Audétat (dir.), 2015, Sciences et technologies émergentes, Pourquoi tant de promesses?, Herman, Paris.

39. Jacobsson et Johnson, 2000.

40. Adamson, 2007.

41. The Scottish Government, Scotland's Economy. The Case for Independence, Edinburgh, 2013.

42. The Scottish Government, 2013a.

43. Seuls 17,9 MW étaient installés en 2017. Source: Digest of UK Energy Statistic 2018, Regional Statistics 2003-2017: Installed Capacity. 
capacity to be developed, agendas to be refined and interdependencies between actors to emerge $e^{44}$.

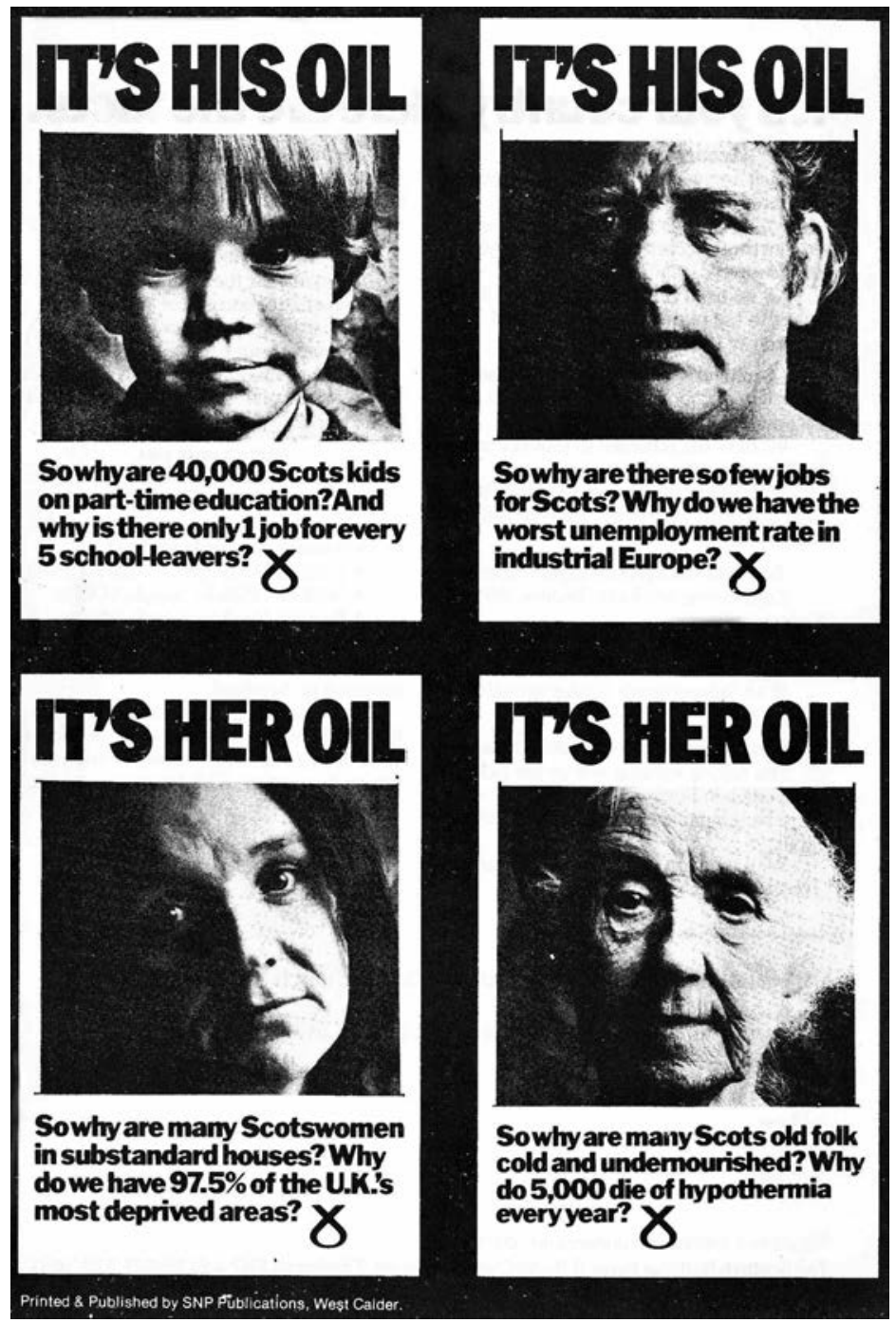

Fig. 1. - « It's his oil, it's her oil »

Brochure du SNP, 1972.

Crédit : Scottish Political Archive, http://www.scottishpoliticalarchive.org.uk/

44. Cowell et al., 2017, p. 177. 
Sur un plan politique, la volonté de développer les énergies renouvelables en Écosse s'inscrit pleinement dans la " conversation nationale " (The National Conversation) engagée par le SNP dès son arrivée au pouvoir en $2007^{45}$. Le recours à des éléments tirés du milieu naturel sert à justifier le projet national écossais, organisé autour de ressources naturelles exceptionnelles. Comme mentionné en 2013 dans le livre blanc pour l'indépendance (Scotland's Future) : "Scotland is blessed with an abundance of natural resources that can bring prosperity and ensure our nation meets the global challenges of the $21^{\text {st }}$ century ${ }^{46}$. " Dans cette optique, le SNP mentionnait son intention d'inscrire dans une constitution l'utilisation des ressources naturelles dans une perspective de développement durable : «Scotland's natural resources are vital to the future success of an independent Scotland ${ }^{47}$. " Le SNP va en effet défendre la vision d'une Écosse écologique (dans une approche en termes de " modernisation écologique "), inspirée par le dynamisme des petits pays sociaux-démocrates nordiques. Le modèle à suivre, qui était celui de la Norvège dans les années 1970 (pays voisin qui gérait ses ressources pétrolières de façon indépendante), devient celui du Danemark ${ }^{48}$ : un pays de taille et de population similaire à l'Écosse, souverainement indépendant au sein de l'Union européenne (UE) mais économiquement favorisé par son intégration au marché unique, berceau du développement de l'éolien dans les années 1980 et modèle de référence en termes de transition énergétique ${ }^{49}$. À ceci, nous pouvons dire que le SNP souhaite faire de l'Écosse une "éco-nation " d'inspiration scandinave si on se réfère à la définition proposée par Tudi Kernalegenn : «Un pays avec une identité culturelle, géographique et politique qui aspire au maximum d'autosuffisance possible dans le contrôle de ses ressources naturelles et dans le cadre d'une Europe des peuples ${ }^{50}$."

Dans son article The Greening of Nationalism : Nationalising Nature in Europe, Paul Hamilton (2002) montre comment les nationalistes écossais ont récupéré le discours environnemental dans les années 1990 pour renforcer et crédibiliser leurs revendications d'indépendance. Ce discours

45. Scottish Executive, 2007.

46. The Scottish Government, 2013a, p. 277.

47. The Scottish Government, 2013b, p. 9.

48. Campbell et Hall, 2009.

49. Les îles Orcades s'inspirèrent des expériences menées au Danemark sur l'éolien pour construire le projet EMEC, notamment celles menées au sein du laboratoire national Risø.

50. Kernalegenn, 2016, p. 8. 
écologique du SNP peut ainsi se voir comme un réalignement de son discours politique avec un nouveau référentiel écologique ${ }^{51}$. Ce discours nationaliste écologique s'inscrit à travers un phénomène d'" environnementalisation " généralisé de l'ensemble des filières énergétiques à l'échelle mondiale. L'énergie est rattachée à des valeurs promotionnelles différentes de celles des années 1950 à 1980. Le discours s'inscrit de plus dans un environnement où l'enjeu énergétique est de mieux en mieux perçu par la population écossaise ${ }^{52}$. Comme le souligne en 2005 le Président de la Royal Society of Edinburgh Sir Michael Atiyah :
Energy issues have become a matter of global concern and in Scotland there has been a rapid escalation of public and political interest. We are at a turning point in our history as we move from being a self-sufficient exporter of energy to a possible dependence on imported energy from an increasingly competitive World ${ }^{3}$.

Pour Dominic Hinde (2016), les années 2008 à 2016 ont vu ainsi se développer un "self-consciously Scottish environmental framework ». La dévolution de $1999^{54}$ dote Edimbourg d'un pouvoir de décision en matière environnementale, et donc par effet de domino, d'un pouvoir de décision en matière d'énergies renouvelables ${ }^{55}$. Selon Heffron et Nuttall (2017):

\section{Despite the formal power of Westminster and Whitehall over Scottish energy policy, the reality, especially as relates to new infrastructure investment, is that the Scottish Government has power over all decision making relating to environmental permission and as such it has a veto over all proposed investments. [...] Hence, while developing its own environmental policy maybe a limited exercise, Scotland has in effect the capability to pursue its own energy policy.}

Les énergies renouvelables vont être le symbole de cette conversion nationale écologique portée par le SNP. Elles vont être présentées comme « une

51. Spaven, 1991.

52. Davidson et al., 2009.

53. The Royal Society of Edinburgh, 2006, p. 1.

54. La dévolution est le processus de réforme décentralisatrice qui a renforcé l'échelon intermédiaire entre l'État et la commune. Le Scotland Act de 1998 instaure un gouvernement local disposant de larges compétences internes et fixe à 129 le nombre des députés au nouveau Parlement écossais dont 56 seront élus à la proportionnelle. https://www.gov.uk/guidance/devolution-of-powers-toscotland-wales-and-northern-ireland, consulté le 16 mars 2019.

55. Guyet, 2018. 
alternative de politique publique ${ }^{56}$ " pour l'après-pétrole afin d'établir un rapport de force avec Londres, notamment en ce qui concerne le devenir de l'énergie électronucléaire en Écosse qui n'est pas de la compétence des institutions décentralisées : "In light of its potential for renewable power and other clean energy technologies, Scotland is likely to meet its energy needs without nuclear power, and without adding to existing radioactive waste $^{57} . "$

Les ressources pétrolières de la mer du Nord s'épuisant, le SNP fait le pari que les énergies renouvelables pourront ainsi prendre le relais sur le moyen et long terme (notamment pour ce qui concerne la rente financière). Pour le professeur David McCrone, "The SNP is very keen to place itself as a forward-looking movement based on renewables ${ }^{58}$. " Avec le pétrole, les énergies offshore sont la principale arme qui peut donner à l'Écosse les moyens de sa puissance économique et politique sur la scène européenne nourrissant l'imaginaire identitaire. Ainsi si dans les années 1970, le nationalisme écossais revendiquait que l'Écosse « possédait » jusqu’à $90 \%$ des réserves pétrolières de la mer du Nord, en 2010, il revendique « le quart de toute la ressource énergétique renouvelable en Europe ${ }^{59}$ » faisant de l'Écosse "l'Arabie Saoudite des énergies marines ${ }^{60}$ ». L'imaginaire du pétrole apparaît ici comme une composante majeure du discours nationaliste écossais. Il s'agit de légitimer le développement des énergies offshore en les inscrivants dans un passé vénérable, celui du mythe de l'abondance énergétique et des richesses nationales, symbolisé par le puits de pétrole. Une phrase de Niall Stuart (directeur de l'organisme Scottish Renewables chargé de promouvoir les énergies renouvelables) prise dans la presse française semble ici bien illustrer cette transition : "Dans les années 1970, les indépendantistes avaient adopté le slogan "c'est le pétrole de l'Écosse". Aujourd'hui, ils devraient dire "c'est le temps de merde de l'Écosse" ${ }^{\text {. }}$."

56. En reprenant la formule d'Aurélien Evrard (2014).

57. Scottish Executive, 2007, p. 14.

58. Entretien avec le professeur David McCrone, Professeur émérite de sociologie à l'Université d'Edimbourg, le 15 mai 2018.

59. Dans son allocution du Nouvel An en 2013, Alex Salmond insista sur le fait que « les ressources de l'Écosse en énergie éolienne en mer, en énergie des courants et en énergie houlomotrice totalisaient environ le quart de toute la ressource énergétique renouvelable en Europe ».

60. "SNP hopes a new wave can carry Scotland to independence ", The Guardian, 29 mai 2011.

61. "L'île d'Eigg, force de la nature », Libération, 24 juin 2013. 
D’un côté ce nationalisme énergétique cherche à s'inscrire dans un héritage historique (celui du pétro-nationalisme) qui lui permet de véhiculer un aspect rassurant. D’un autre côté, il cherche à se démarquer de cet héritage en promouvant la rupture et la nouveauté sous l'angle modernisateur de la transition écologique. Les énergies renouvelables offshore permettent ainsi de moderniser un nationalisme vieillissant représenté par l'industrie du pétrole en lui collant le projet de transition énergétique. S’exprimant à Washington DC en 2008, le Premier ministre écossais Alex Salmond déclarait :

40 years ago, with the discovery of oil in the North Sea off Scotland's coast, Scotland won the natural lottery [...] And we are fortunate that Scotland's energy resources go far beyond hydrocarbons [...] Our powerful waters and our driving offshore winds are key to our future prosperity [...] We have won the natural lottery once again ${ }^{62}$.

\section{Moderniser le nationalisme énergétique par le projet libéral européen}

Le " nationalisme civique " que prône le SNP participe à la définition d'une identité politique qui privilégie le contrôle des leviers économiques et politiques nécessaires à la gestion d'une Écosse indépendante ${ }^{63}$. Il se veut intégrateur, ouvert à la coopération et tourné vers l'Europe depuis la fin des années 1980, symbolisé par le slogan "Scotland in Europe " ${ }^{64}$. En citant Gilles Leydier : «Pour les nationalistes écossais, l'Union européenne constitue un cadre de référence alternatif à l'État britannique, attractif en termes d'opportunités économiques, de sécurité collective, de reconnaissance internationale et de promotion d'un agenda social-démocrate ${ }^{65}$. $\gg \mathrm{Ce}$ discours pro-européen prend la tournure d'un outil stratégique permettant aux nationalistes de légitimer leur désir d'autonomie au sein de l'Union européenne, tout en crédibilisant leur projet d'indépendance. Le développement de l'Europe des régions a en effet encouragé la mise en place de

62. Alex Salmond, Renewable Energy : Innovations that Can Save ou Planet, Discours à la National Geographic Society (NGS), Washington DC, 2 avril 2008. https://www2.gov.scot/News/ Releases/2008/04/02151533, consulté le 23 avril 2019.

63. Fiasson, 2016.

64. Ichijo, 2004.

65. Leydier, 2016, p. 60. 
conditions particulièrement propices à l'émergence des " nationalismes régionaux ${ }^{66}$. "

L'intégration européenne représente ainsi une structure d'opportunités pour les nationalistes, fournissant des ressources inédites pour renforcer leurs positions vis-à-vis des administrations londoniennes ${ }^{67}$. La montée en puissance des énergies renouvelables en Écosse n'est pas étrangère à la profonde restructuration du secteur énergétique au sein de l'Union européenne ${ }^{68}$. La mise à l'agenda politique s'opère en effet dans le cadre d'un modèle contraint par des directives européennes en matière d'énergies renouvelables, couplées à une politique volontariste de Londres sur ce même sujet ${ }^{69}$. Cette dynamique européenne va encourager les nationalistes à positionner l'Écosse comme acteur avant-gardiste et "bon élève " de l'Europe, participant à la création d'un leadership climatique écossais à l'échelle européenne : «Scotland could play a leading role in addressing the challenge of climate change and meeting European targets for renewable energy through its potential in wind, tide and wave power ${ }^{70}$. " L'objectif est bien ici de positionner l'Écosse en tant que poumon énergétique de l'Europe (couplage pétrole et énergies renouvelables) comme en témoigne la campagne publicitaire "Welcome to Europe's power source ${ }^{71}$ »:

With as much as a 25\% of Europe's offshore wind and tidal potential, an estimated $10 \%$ of its capacity for wave power and its largest offshore $\mathrm{CO}_{2}$ storage potential, Scotland has the natural resources to become the green energy powerhouse of Europe ${ }^{72}$.

66. Tétart, 2010.

67. Alexandre-Collier, 2006.

68. Veyrenc, 2010.

69. Le Royaume-Uni est un pays avant-gardiste dans le domaine de la transition énergétique du fait de l'adoption précoce d'un engagement législatif à long terme pour un avenir à faible émission de carbone. Une cible de carbone a été définie explicitement en 1990, avec l'engagement de réduire les émissions de carbone au niveau de 1990 en 2005. L'une des initiatives de transition les plus ambitieuses reste néanmoins le Climate Change Act de 2008 qui engage le pays sur un objectif de réduction spectaculaire de $80 \%$ des émissions de gaz à effet de serre entre 1990 et 2050 (Lockwood, 2013). Aussi, en lien avec l'accord européen sur le " paquet énergie-climat » de 2008, le RoyaumeUni s'engage à faire passer à $15 \%$ la part des énergies renouvelables dans la consommation totale du pays à l'horizon 2020 (contre $1,3 \%$ en 2005).

70. http://www.gov.scot/Publications/2009/11/26155932/7, consulté le 29 avril 2019.

71. https://www.scotland.org/features/welcome-to-europes-power-source, consulté le 29 avril 2019.

72. The Scottish Government, 2011, p. 10. 
En 2004 est construit dans les Orcades l'European Maritime Energy Centre (EMEC), un organisme entièrement dédié aux nouvelles énergies de la mer. Celui-ci se positionne immédiatement à l'échelle européenne afin de séduire les firmes étrangères, obtenir des subventions et pour renforcer l'aura internationale du site d'essais, témoignant de la volonté de l'archipel de bien s'insérer dans les réseaux européens ${ }^{73}$.

Il est enfin important de noter que la conception dite " civique » du nationalisme écossais (un nationalisme qui se veut ouvert et intégrateur) s'investit dans la manière qu'ont les Écossais d'appréhender leur politique énergétique. Là où la France a fait le choix de passer par ses " champions nationaux " pour réaliser sa transition énergétique dans une vision colbertiste (pensons ici à l'ambitieux plan solaire porté par EDF qui vise à développer $30 \mathrm{GW}$ d'énergie solaire photovoltaïque en France métropolitaine d'ici 2035), l'Écosse (et plus largement le Royaume-Uni) a fait le choix d'encourager l'implantation d'entreprises étrangères (par des mesures fiscales avantageuses), tout en continuant à mettre sous les projecteurs ses petites pépites nationales "made in Scotland" au destin plus ou moins contrarié (comme la startup Pelamis Wave Power dans l'énergie des vagues ou Scotrenewables dans l'hydrolien). Le nationalisme énergétique s'inscrit donc dans un "modèle économique franchement libéral, qui met l'accent sur la productivité et la compétitivité des entreprises, le développent des nouvelles technologies, la recherche de produits innovants, le dynamisme à l'exportation et l'accueil des investissements étrangers " (Leydier, 2006). Dans son ouvrage L'investissement étranger, moteur de la réindustrialisation au Royaume-Uni ?, Louisa Toubal (2018) nous rappelait que les investisseurs étrangers occupent depuis très longtemps une place particulière au Royaume-Uni pour soutenir l'industrie, notamment après les réformes de Margaret Thatcher. Londres encourage en effet les régions et les territoires locaux à mettre en place leurs propres stratégies pour attirer les investissements directs à l'étranger (IDE) ${ }^{74}$. Cette ouverture aux IDE doit permettre de reconvertir l'économie, de nourrir la concurrence, de développer l'emploi, d'attirer les salariés qualifiés, de faire foisonner les projets et d'accélérer la baisse des coûts des technologies par des économies d'échelle. Le discours ne vise donc pas à nationaliser l'économie en appliquant des

73. http://www.emec.org.uk/europes-world-leading-technologies-showcased-in-orkney/, consulté le 29 avril 2019.

74. Bailoni, 2011. 
mesures protectionnistes. Il ne prend pas la tournure d'un protectionnisme nationaliste excluant, comme on peut le voir dans certaines formes de pétro-nationalismes ${ }^{75}$.

La stratégie écossaise vise donc essentiellement à valoriser des compétences clefs d'une industrie hautement internationalisée (celle du pétrole) vers une industrie périphérique émergente (celle des énergies offshore), l'objectif étant de trouver des complémentarités (cross-sector opportunities) entre une industrie ancienne et une industrie émergente ${ }^{76}$, afin de positionner l'Écosse comme un territoire leader ${ }^{77}$.

The skills and expertise which have made the North Sea oil and gas industry such a world-class success can be harnessed to drive the renewable sector [...] The Scottish Government is confident that the knowledge and expertise from oil and gas will be a huge competitive advantage to the marine industry of renewable technologies ${ }^{78}$.

Scotland has long been an energy-rich nation. That reputation, forged in the development of our coal, oil and gas reserves and engineering prowess, has since grown through the rapid development of our renewable resource $e^{79}$.

L'Écosse compte ainsi aujourd'hui s'appuyer sur l'expertise des multinationales du oil and gas pour se positionner comme pays leader de l'éolien flottant, à l'instar du groupe norvégien Statoil qui développe en Écosse le "plus grand parc éolien flottant au monde ${ }^{80}$ ". Cette entreprise expérimente aussi le stockage d'énergie sur batteries appliqué à l'éolien en mer, ce qui constitue là aussi une première à l'échelle mondiale. Ces implantations d'entreprises étrangères se caractérisent soit par la création de filiales ou d'usines en Écosse (greenfield investments), soit par le rachat de firmes ou de projets préexistants (brownfield investments). L'acquisition en mai 2018

\footnotetext{
75. Stevens, 2008.

76. Selon une étude de Scottish Enterprise (2017), la diversification de l'industrie du oil and gas serait directement profitable à 24 secteurs, dans l'éolien en mer et les énergies marines.

77. Taylor, 2017.

78. The Scottish Government, 2013c, p. 20.

79. The Scottish Government, 2017, p. 17.

80. "Malmené par la chute du pétrole, le norvégien Statoil s'essaie aux renouvelables ", Les Échos, 27 avril 2017.
} 
d'un projet de parc éolien en mer par EDF au large de l'Écosse est symbolique de ce choix industriel ${ }^{81}$.

À Aberdeen, capitale européenne du pétrole, le centre européen de déploiement éolien offshore (The European Offshore Wind Deployment Centre) et ses onze éoliennes marines marque dans le paysage la transition de l'Écosse vers les énergies offshore ${ }^{82}$. Ce lien entre héritage (le pétrole) et le futur (les énergies renouvelables) se retrouve dans le musée maritime d'Aberdeen (Aberdeen Maritime Museum). Bien que l'épopée des hydrocarbures soit retracée, plantée au pied des remorqueurs et ravitailleurs de l'industrie pétrolière, la transition vers les énergies renouvelables offshore est mentionnée (Fig. III et IV, cahier couleur). Selon un élu SNP d'opposition à la municipalité d'Aberdeen : "Le musée maritime d'Aberdeen permet de montrer à la population ce qu'on a fait dans le pétrole, et ce que l'Écosse peut faire désormais faire dans le secteur des énergies de l'océan ${ }^{83}$ ".

\section{Faire rayonner l'Écosse par les nouvelles technologies énergétiques}

Ce nationalisme énergétique alimente les fantasmes d'un pays autonome transformé par les nouvelles technologies de l'énergie. Si après la guerre, la technique nucléaire devint « la quintessence même du symbole de la modernité et de la puissance nationale ${ }^{84}$ ", le $\mathrm{XXI}^{\mathrm{e}}$ siècle est présenté comme une course internationale autour des nouvelles technologies environnementales $^{85}$, notamment énergétiques ${ }^{86}$. En prenant la Chine comme exemple, Richard Balme et Giulia Clara Romano (2014) nous montrent comment l'énergie s'inscrit au cœur des politiques de modernisation des Etats. Le caractère innovant des énergies offshore permet au discours nationaliste de présenter l'Écosse comme un pays moderne et à la pointe des nouvelles technologies :

81. «EDF acquiert un gros projet de parc éolien en mer en Écosse », Le Figaro, 3 mai 2018.

82. "Des éoliennes offshores de 8,8 MW en service en Écosse ", Connaissance des énergies, 12 juillet 2018.

83. Entretien avec Christian Allard, élu SNP à la municipalité d'Aberdeen, le 30 mai 2018.

84. Hecht, 2014, p. 11.

85. Glachant, 2014.

86. Therme, 2011. 
Scotland's extraordinary natural marine energy resources and leading position in the development of wave and tidal energy technologies provide a unique platform to establish a world leading position in this vitally important sector ${ }^{87}$.

Le rayonnement national s'exprime ici d'abord en termes de prouesses technologiques, l'objectif étant de présenter le programme indépendantiste comme stratège et intelligent (par ses entreprises de hautes technologies et sa recherche-développement), afin de crédibiliser les capacités d'autonomie économique du pays. On rejoint ici la thèse présentée par Nicolas Mazzucchi pour qui « les choix de certaines énergies révèlent des ambitions d'affirmation de l'image nationale par la possession de technologies avancées (ENR, nucléaire ${ }^{88} »$. En examinant l'évolution de la stratégie électronucléaire du gouvernement du Québec entre 1963 et 2012, Mahdi Khelfaoui (2014) montre ainsi comment l'option électronucléaire s'est présentée dans les années 1960 comme un symbole de rayonnement technologique et de prospérité économique aux yeux des décideurs politiques, ainsi qu'un outil privilégié pour diffuser des discours nationalistes.

162 Ce nationalisme se nourrit enfin de la vision d'un peuple écossais particulièrement ingénieux qui valorise la figure de l'innovateur héroïque et les grandes réussites technologiques nationales ${ }^{89}$. Pour Arnaud Fiasson : "Lidentité écossaise proposée par le SNP ne repose pas sur une définition exclusivement culturelle : elle relève de l'exploitation des ressources humaines et matérielles au sein de l'entité géopolitique que représente l'Écosse ${ }^{90}$. "Ce nationalisme, qui jette un fil rouge historique entre un passé écossais mythologisé et un avenir désiré qu’est la transition énergétique, est largement relayé par une importante audience médiatique :

\section{In this period of economic uncertainty, I would still bet the political} and economic house on a global renewables transition. It was James Blyth, a Scottish inventor, who made the first prototype wind turbine in 1887, a century after the Scottish engineer James Watt built his

87. The Scottish Government, 2010, p. 48.

88. Mazzuchi, 2017, p. 156.

89. Herman, 2003.

90. Fiasson, 2016, p. 12. 
steam engine to power the world. Scottish innovation and industry are once again leading an energy revolution ${ }^{91}$.

Cette culture de l'ingénieur définit l'identité nationale écossaise qui permet aux individus d'imaginer ce qui fait le caractère distinctif et singulier de leur pays (les ressources naturelles et les ingénieurs qui tentent de les valoriser par l'innovation technologique). Pour la professeure Edwige Camp-Pietrain : «Dans tous les discours, on retrouve année par année ces références à ces grandes figures historiques. Elles permettent de distinguer l'Écosse dans le Royaume-Uni ${ }^{92}$. " Les symboles du nationalisme énergétique sont aisément reconnaissables et ont prouvé leur capacité à rallier des points de culture populaire à travers les grands personnages historiques :

\section{James Watt's initial is stamped on every light bulb, measuring the} power it delivers, but also reminding us of the intellectual light and sheer progress he brought to the world. Rather than obsess over the symbolism of divestment, this is what we need to invest in ${ }^{93}$.

Ce nationalisme énergétique s'inscrit dans une culture d'ingénieur qui est valorisée en Écosse depuis au moins le $\mathrm{XIX}^{\mathrm{e}}$ siècle, en partie car il permettait à des Écossais une mobilité sociale au sein du Royaume-Uni et de son empire (Houston et Withers, 1990). Elle permet aussi de jouer sur les rivalités avec les ingénieurs anglais. En citant Ross et al.:

The process of recapture is represented by an aspiration to recover historically positive features of national identity now regarded as problematic [...] The perception of Scotland as a place with an entrepreneurial and innovative history usually takes the form of references to the large Scottish commercial empires of the past, or, more commonly, the citation of the many famous' Scots inventors and innovators ${ }^{94}$.

Présenté à Washington en 2008, le Saltire Prize ${ }^{95}$ est le prix national récompensant les avancées technologiques dans le développement commercial des énergies offshore. Il se veut alors le plus grand prix jamais attribué au monde pour l'innovation dans le secteur des énergies marines renouve-

91. "Scottish innovation is leading a global energy revolution », The Times, 22 décembre 2017.

92. Entretien avec Edwige Camp-Pietrain, Professeur de civilisation britannique à l'Université de Valenciennes, le 23 avril 2018.

93. Colin McInnes, 2005.

94. Ross et al., 2003, p. 380.

95. Le drapeau de l'Écosse est également connu sous le nom de croix de Saltire. 
lables. S'exprimant sur le sujet, le ministre écossais Alex Salmond déclarait en 2008 :

The Saltire Prize is the Scottish Government's way of playing its part in inspiring a revolution in clean, green energy as the world enters a new golden age in innovation prizes [...] Scotland has long been a leader in scientific advances and is ranked second per head of population in the world behind only Switzerland and ahead of the USA in terms of impact of the research we produce [...] With demonstration of the ideas in Scotland, the Saltire Prize will deliver clear economic benefits at home as well as pushing forward the boundaries of research with a world-wide impact and benefit ${ }^{96}$.

Les îles écossaises vont pleinement participer à la diffusion de la technopolitique en se positionnant rapidement comme des vitrines technologiques de l'Écosse : "The development of renewables on the Scottish Islands [...] is an opportunity to establish Scotland as a world leader in marine technologies ${ }^{97}$. " Riches de ressources naturelles inépuisables, les îles écossaises se présentent comme des laboratoires à ciel ouvert pour tester de nouvelles technologies énergétiques ${ }^{98}$. Le lancement de projets énergétiques innovants permet en effet aux îles à la fois d'avancer dans des projets autonomistes post-pétrole ${ }^{99}$, et en même temps, de trouver une forme de reconnaissance médiatique et politique à travers la modernisation intellectuelle et technologique. Parmi ces nombreuses îles, les Orcades et son laboratoire EMEC sont devenus le porte-drapeau à l'international de cette Écosse qui se veut écologique, dynamique et innovante. Parler d'énergies marines au Royaume-Uni, c'est en effet immanquablement associer l'Écosse et son archipel des Orcades. La technopolitique des énergies marines s'exprime territorialement dans une dimension insulaire (nous invitons à réfléchir ici au concept de "techno-insularisme »), ces technologies n'en finissant pas d'être présentées comme salvatrices par la presse britannique, pour une région désormais érigée comme modèle en matière

96. Alex Salmond, Renewable Energy : Innovations that Can Save ou Planet, Discours à la National Geographic Society (NGS), Washington DC, 2 avril 2008. https://www2.gov.scot/News/ Releases/2008/04/02151533, consulté le 23 avril 2019.

97. Scottish Islands Renewable Project, Department of Energy \& Climate Change, 2013.

98. L'Écosse possède plus de 790 îles regroupant 103702 résidents permanents (recensement de 2011).

99. Johnson et al., 2013. 
de transition énergétique : "If you want to know what Scotland could look like in 10 years then take the 90-minute ferry journey from Scrabster to Stromness and find out ${ }^{100}$ "; "Islanders set sail on pollution-free ferries project ${ }^{101}$ "; "Tidal energy site in Orkney in hydrogen "first" 102 »; "How Orkney leads the way for sustainable energy ${ }^{103} »$. Dans les îles écossaises, le développement de projets énergies marines est légitimé par un discours autonomiste et communautaire ${ }^{104}$.

Le nationalisme s'exprime aussi au cœur de la capitale écossaise. Une section est ainsi consacrée aux énergies marines au Musée National d'Écosse (National Museum of Scotland) à Edimbourg (Fig. V et VI, cahier couleur). La présentation est brève mais riche en explications : le visiteur apprend que l'énergie des mers est un secteur dans lequel les Ecossais sont pionniers et s'exercent avec succès depuis plusieurs décennies. En 1974, le papier Waver power de l'écossais Stephen Salter dans la revue Nature (Volume 249) fait entrer définitivement l'énergie des vagues dans la sphère scientifique. De nombreux mécanismes sont alors proposés, testés ou même réalisés à l'échelle $1 / 1$, le plus connu étant les fameux Canards de Salter ${ }^{105}$ (Salter's Ducks), mis au point à l'Université d'Edimbourg. Ces projets pionniers vont permettre de positionner les universités écossaises comme des références internationales sur la technologie des vagues (wave energy). L'histoire écossaise tient ainsi un nouvel héros national dans les technologies énergétiques " made in Scotland, changing the world ${ }^{106}$ ", poursuivant un dialogue vénérable et mythifié entre passé et avenir ${ }^{107}$.

100. «Orkney Islands provide a glimpse of a renewable future », The Guardian, 20 septembre 2011. 101. The Times, 6 août 2017.

102. BBC News, 13 septembre 2017.

103. The Guardian, 20 janvier 2019.

104. Roche, 2019.

105. Le professeur Stephen Salter a reçu la première médaille Saltire Prize en 2011.

106. https://www.nms.ac.uk/explore-our-collections/stories/science-and-technology/made-inscotland-changing-the-world/, consulté le 24 avril 2019.

107. Ainsi dans la partie du National Museum of Scotland intitulé "Innovating Scots ", il est expliqué aux visiteurs : "Scot have a long history of innovation and invention. The have made ground-breaking discoveries and promoted ideas that have changed lives and helped shape de modern world. Scottish men and women have made outstanding contributions in medecine, mathematics, education, engineering, exploration, communications and many others areas of human endeavour ". 


\section{Conclusion : la rupture dans la continuité ?}

La trajectoire écossaise dans le domaine des énergies marines est sans commune mesure avec celles des autres pays européens. L'Écosse a su s'établir médiatiquement comme un des leaders dans ce secteur émergent en profitant au maximum des avantages que procurent ses ressources naturelles. Pour cela, elle s'est appuyée sur de nombreux outils de valorisation, notamment sa position de leader européen dans l'industrie du pétrole. Cette identification est entretenue par un écosystème d'innovation liant des acteurs publics, privés et associatifs, travaillant de concert sur le développement des énergies renouvelables. L'exemple des énergies renouvelables offshore nous montre aussi qu'elle est intimement liée à la modernité technologique. Le nationalisme écossais ne s'investit pas seulement dans le rugby ou le tartan mais aussi dans la mer et le vent. Les énergies renouvelables offshore étant à la croisée du nationalisme des ressources naturelles et du techno-nationalisme, elles ne pouvaient qu'acquérir un statut particulier au sein d'une nation qui a fait de l'énergie un marqueur identitaire fort de son histoire. Leur développement s'intègre dans une stratégie nationaliste qui considère la politique énergétique comme un élément central de l'avenir économique d'une Écosse indépendante ${ }^{108}$. Ces technologies sont en effet inscrites dans un discours indépendantiste qui participe à la réinterprétation historique du premier " mythe » énergétique écossais, celui de l'exploitation du pétrole offshore, dans un discours renouvelé et modernisé (par le projet libéral, européen et écologique), puisque porté cette fois sur le développement d'énergies vertes (énergie éolienne offshore ou marémotrice). En citant Nicola McEwen et Elizabeth Bomberg (2014):

\section{What is intriguing about the Scottish case, however, is that it includes an embrace of both 'old' and 'new' energy: renewable energy and emissions reductions are pursued alongside the evident desire to reap the rewards (following independence) of North Sea oil.}

"La science contribue avec des éléments neufs, revisités, à reconstituer des mythes anciens " écrivait le chimiste Paul Caro en 1994. Il est certain que cette thèse se confirme en Écosse dans le domaine des énergies offshore. 
L'historien Martin Pâquet utilise ainsi le concept "d'usage du passé » pour définir " une série de pratiques - pratiques rhétoriques ou discursives, mais aussi symboliques, catégorielles, classificatrices ou commémoratives - faisant référence à des représentations sociales d'un passé proche ou lointain ${ }^{109}$ ". À ceci Bernadette Bensaude-Vincent rajoute que «le passé historique est souvent instrumentalisé pour crédibiliser et légitimer une ligne d'action présente ${ }^{110}$ ».

L'invocation de la nation et d'un passé mythologisé (celui de James Watt et du mythe de l'abondance pétrolière) doit ici permettre de créer un sentiment d'objectivité qui à son tour doit participer au travail de légitimation des énergies offshore. D’un côté, les nationalistes écossais cherchent à inscrire les énergies en mer dans un héritage historique, qui est celui d'une nation qui a toujours cherché à valoriser autant que possible ses ressources naturelles (le charbon, le pétrole et maintenant les énergies renouvelables). D’un autre côté, ils cherchent à les démarquer de cet héritage à travers un projet écologique et européen pour promouvoir la nouveauté et l'ouverture. Les énergies offshore ont ainsi réussi à établir une continuité avec un discours nationaliste approprié.

Innovation (though not necessarily successful commercialisation in the past) is Scotland's technological identity. The oil and gas industry, maritime heritage and marine expertise (as well as natural waveltidal resource) are all key drivers of energy policy ${ }^{111}$.

Nous devons néanmoins souligner que le développement des énergies offshore en Écosse ne répond pas forcément à la même logique : le déploiement de parcs offshore dans la région pétrolifère d'Aberdeen signifie autre chose comparé aux petites unités de production (wave and tidal) développées dans les territoires insulaires périphériques. Et à ce titre, nous insistons sur le fait que le discours nationaliste des énergies offshore ne prend pas la même forme et dimension en fonction des technologies. D’un côté nous retrouvons l'éolien en mer, technologie maitrisée et centralisée, qui est entrée dans une phase opérationnelle dès les années 1990 au Royaume-Uni. Cette technologie s'inscrit dans une dynamique très business et capitalistique, et à ce titre elle répond avant tout à un discours nationaliste libéral, dans la mesure où son développement est confié

109. Pâquet, 2006, p. 175.

110. Bensaude-Vincent, 2015, p. 65.

111. Entretien avec Jane Watters, Responsable innovation à Scottish Enterprise, le 4 mai 2018. 
à de grandes multinationales de l'énergie. Rajoutons à ceci que le déploiement de l'éolien marin reste une politique de production d'énergie pilotée avant tout par les institutions londoniennes. C'est bien Londres qui fixe les grandes orientations stratégiques pour les années à venir ${ }^{112}$. Les chiffres témoignent que la dynamique de l'éolien offshore au Royaume-Uni est anglaise avant d'être écossaise ${ }^{113}$.

D'un autre côté, nous trouvons des technologies plus innovantes et moins matures (type hydrolien ou houlomoteur), inscrites dans une dynamique beaucoup plus $\mathrm{R} \& \mathrm{D}$ et académique, relevant de la décentralisation énergétique et de la petite production d'électricité. Ces deux filières technologiques répondent avant tout à une politique d'innovation, et à ce titre, elles sont volontairement inscrites dans un discours techno-nationaliste qui valorise les savoir-faire scientifiques et techniques et glorifie les grandes figures écossaises (à l'image de l'EMEC et du professeur Stephen Salter par exemple $)^{114}$. Comme le mentionnent Toke et al. : "Support for the nascent wave and tidal stream industries has been the bedrock of the Scottish government's enthusiasm for renewable energy development ${ }^{115}$. "

168 Dans les faits, nous défendons donc l'idée que le nationalisme écossais s'exprime essentiellement dans une politique d'aide à l'innovation (wave and tidal) et non dans une politique de production massive d'énergie verte (offshore wind) qui reste sous la responsabilité de Londres ${ }^{116}$. Aussi, s'il est certain que nous pouvons parler de l'existence d'un techno-nationalisme écossais assumé pour le wave and tidal (s'appuyer sur des technologies innovantes pour porter un discours d'affirmation national), parler d'un techno-nationalisme écossais pour le offshore wind nous paraitrait ici excessif et critiquable.

112. La ministre britannique de l'Énergie et de la Croissance verte, Claire Perry, a dévoilé, le jeudi 7 mars 2019, le contrat de filière pour l'éolien en mer. Le Royaume-Uni, qui veut rester le premier marché européen en la matière, vise une capacité de 30 GW d'ici 2030, ce qui représenterait un cinquième des capacités mondiales. Porter la part de l'éolien en mer à plus de $30 \%$ permettrait aux énergies renouvelables dans leur ensemble de peser plus lourd que les énergies fossiles dans la production d'électricité. https://www.gov.uk/government/news/offshore-wind-energy-revolutionto-provide-a-third-of-all-uk-electricity-by-2030, consulté le 24 avril 2019.

113. En 2017, la capacité offshore anglaise s'élevait à 6015 MW, pour une capacité écossaise de 246 MW. Digest of UK Energy Statistic 2018, Regional Statistics 2003-2017: Installed Capacity: https://www.gov.uk/government/statistics/regional-renewable-statistics, consulté le 26 avril 2019.

114. https://www.scotland.org/business/conferences-and-meetings/legends/technology/technology-scotland, consulté le 26 avril 2019.

115. Toke et al., 2013, p. 61.

116. «Scottish government launches $£ 10 \mathrm{~m}$ Saltire Fund », BBC News, 10 février 2019. 


\section{Bibliographie}

Adamson Kerry-Ann, Stationary Fuel Cells: An Overview, Elsevier, Oxford, 2007. Adria Marco, Technology and Nationalism, McGill-Queen's University Press, 2010.

Aкrich Madeleine, "Comment décrire les objets techniques ?", Techniques \& Culture, no 9, 1987, p. 49-64.

Alexandre-Collier Agnès, "L'instrumentalisation de l'Union européenne face à la dévolution ", Revue Française de Civilisation Britannique, XIv-1, 2006, p. 43-56.

BAILONI Mark, "Les évolutions du modèle énergétique britannique face aux enjeux géopolitiques internes ", VertigO - la revue électronique en sciences de l'environnement, vol. 4, $\mathrm{n}^{\circ} 3$, décembre 2014, URL : http://journals.openedition. org/vertigo/15550; DOI : 10.4000/vertigo. 15550 .

BAILONI Mark, "Les investissements étrangers au Royaume-Uni : recomposition des territoires, rivalités géopolitiques et contrecoups identitaires ", L'Espace politique, 15, 2011, URL : http://journals.openedition.org/espacepolitique/2084; DOI : 10.4000/espacepolitique.2084.

Balme Richard, Romano Giulia Clara, «La politique énergétique au cœur de la modernisation chinoise ", Revue française d'administration publique, vol. 150, $\mathrm{n}^{\circ} 2$, 2014, p. 435-452.

BARTHE Yannick, "Les qualités politiques des technologies. Irréversibilité et réversibilité dans la gestion des déchets nucléaires ", Tracés, 2009, p. 119-137.

Bellavance Claude, "Les origines économiques et techniques de la nationalisation de l'électricité au Québec : l'expérience du régime mixte, de 1944 à 1963 ", Annales historiques de l'électricité, nº 1, 2003, p. 37-52.

Bensaude-Vincent Bernadette, "Promesses et régime d'historicité en technosciences ", dans Sciences et Technologies émergentes: Pourquoi tant de promesses?", Hermann, Paris, 2015, p. 49-68.

Bond Ross, Mccrone David, Brown Alice, "National identity and economic development: reiteration, recapture, reinterpretation and repudiation", Nations and Nationalism, 9(3), 2003, p. 371-391.

Bouzarovski Stefan, Bassin Mark, "Energy and Identity: Imagining Russia as a Hydrocarbon Superpower ", Annals of the Association of American Geographers, vol. 101, n 4, Geographies of Energy, 2011, p. 783-794.

Camp-Pietrain Edwige, "Les effets de la crise économique et financière sur les questions constitutionnelles en Écosse ", Revue Française de Civilisation Britannique, 2016 ; DOI : 10.4000/rfcb.959. 
Campbell John L, Hall John A, « National identity and the political economy of small states ", Review of International Political Economy, vol. 16, 2009, p. 547-572.

Caro Paul, "La science, produit de l'imaginaire, fille de l'imagination ", Sciences et imaginaire (dir. I. A. Maréchal), Albin Michel, Paris, 1994, p. 72-80.

Collier Paul, "Ressources naturelles, développement et conflits : liens de causalité et mesures politiques ", Revue d'économie du développement, vol. 12, n 3, 2004, p. 197-215.

Cowell Richard, Ellis Geraint, Sherry-brennan Fionnguala, Strachan Peter A., Toke David, "Energy transitions, sub-national government and regime flexibility: how has devolution in the United Kingdom affected renewable energy development?", Energy Research \& Social Science, 23, 2017, p. 169-181.

Davidson Sara, Martin Chris, Treanor Steven, Scottish Environmental Attitudes and Behaviours Survey 2008, Scottish Government Social Research, 2009.

Debeir Jean-Claude, Deleage Jean-Paul, Hemery Daniel, Une histoire de l'énergie, Flammarion, Paris, 2013.

Edgerton David, Quoi de neuf? Du rôle des techniques dans l'histoire globale, Seuil, Paris, 2013.

Evrard Aurélien, "Les énergies renouvelables et l'électricité. À propos d'un conflit entre un secteur et une alternative de politique publique ", Écologie \& politique, vol. 49, $\mathrm{n}^{\circ}$ 2, 2014, p. 67-80.

FIASsON Arnaud, "Territorialité politique, nationalisme et traversées constitutionnelles en Écosse ", Études écossaises, 18, 2016, URL : http://etudesecossaises.revues.org/1069.

Forchtner Bernhard et KølvraA Christoffer, "The Nature of Nationalism: Populist Radical Right Parties on Countryside and Climate ", Nature and Culture, vol. 10, n², 2015, p. 199-224.

Glachant Matthieu, 2014, "L'innovation et la course aux technologies vertes entre l'Europe et les Pays en développement ", session du séminaire Développement durable et économie de l'environnement à l'IDDRI, 16 juin 2014, Paris.

Goetschel Laurent, Peclard Didier, "Les conflits liés aux ressources naturelles. Résultats de recherches et perspectives ", Annuaire suisse de politique de développement, 25-2, 2006, URL : http://journals.openedition.org/aspd/255.

Hache Emmanuel, "L'OPEP, les compagnies internationales, les compagnies nationales : qui gouverne la scène pétrolière mondiale? ", La Revue de l'Énergie, $n^{\circ}$ 629, 2016.

Hamilton Paul, "Nationalims and Environmentalism", dans Nations and Nationalism: A Global Historical Overview, 2008, p. 875-887. 
Hamilton Paul, "The Greening of Nationalism: Nationalising Nature in Europe”, Environmental Politic, vol. 11, n 2, 2002, p. 27-48.

Hassan Gerry et Barrow Simon, A Nation Changed?, The SNP and Scotland Ten Years On, Luath Press, Edinburgh, 2017.

Неснт Gabrielle, Le rayonnement de la France. Énergie nucléaire et identité nationale après la Seconde Guerre mondiale, La Découverte, Paris, 2014.

Heffron Raphael J., Nuttall William J., "Scotland, Nuclear Energy Policy and Independence", dans Wood Geoffrey et BAKer Keith (dir.), A Critical Review of Scottish Renewable and Low Carbon Energy Policy. Energy, Climate and the Environment, Palgrave Macmillan, Cham, Switzerland, 2017, p. 103-126.

Hinde Dominic, "It's Our Environment: Two Terms of SNP Environmental Policy ", Scottish Affairs, Jan 2016, vol. 25, n 1, 2016, p. 83-102.

Houston Robert Allan, Withers Charles W. J., « Population mobility in Scotland and Europe, 1600-1900: a comparative perspective " dans Annales de démographie historique. Démographie des villes et des campagnes, 1990, p. 285-308.

Jacobsson Staffan, JoHnson Anna, "The diffusion of renewable energy technology: an analytical framework and key issues for research ", Energy Policy, n ${ }^{\circ} 28$, 2000, p. 625-640.

Johnson Kate, Kerr Sandy, Side Jonathan, " Marine renewables and coastal communities. Experiences from the offshore oil industry in the 1970s and their relevance to marine renewables in the 2010s ", Marine Policy, vol. 38, 2013, p. 491-499.

Joris Geoffrey, «Déconstruire le spectacle politique : quand les médias mettent en scène ", Pyramides, n 23, 2012, p. 225-254.

Keating M. J., "Nations against the State: The New Politics of Nationalism in Quebec ", Catalonia and Scotland ( $2^{\mathrm{e}}$ éd.), Basingstoke, Palgrave Macmillan, 2001.

Khelfaoui Mahdi, «Le nucléaire dans la stratégie énergétique du Québec, 19632012 », Scientia Canadensis, n 37(1-2), 2014, p. 105-132.

Latour Bruno, Aramis ou l'amour des techniques, La Découverte, Paris, 1992.

LEYDier Gilles, "Entre libéralisme, social-démocratie et intégration européenne : un modèle écossais ? ", Observatoire de la société britannique, n 1, 2006, p. 235-251.

LEYDier Gilles, "Nationalisme, autonomie et indépendance en Écosse aujourd'hui, dans Autonomies et indépendances : le nationalisme au XXI ${ }^{\mathrm{e}}$ siècle", Connaissances et Savoirs, Saint-Denis, 2016, p. 49-62.

Lockwood Matthew, 2013, "The political sustainability of climate policy: The case of the UK Climate Change Act ", Global Environmental Change, vol. 23, $\mathrm{n}^{\circ} 5$, p. 1339-1348. 
GuYET Rachel, 2018, «Entre centralisation de l'énergie et émergence de nouveaux modèles énergétiques locaux. Le cas d'Aberdeen » dans Lepesan, Gilles (dir.), Énergies nouvelles, territoires autonomes?, Presses de l'Inalco, Paris, p. 137-153.

IcHijo Atsuko, Scottish Nationalism and the Idea of Europe: Concepts of Europe and the Nation, Routledge, Londres, 2004.

Luna Pablo, Mignemi Niccolo (dir.), Prédateurs et résistants, Appropriation et réappropriation de la terre et des ressources naturelles (16e-20 siècles), Syllepse, Paris, 2017.

Mazzucchi Nicolas, Énergie, ressources, technologies et enjeux de pouvoir, Armand Colin, Paris, 2017.

McEwen Nicola, "Energy Policy, Nationalism and Scottish Independence ", dans G. Hassan and J. Mitchell (éd), After Independence: The State of the Scottish Nation Debate, Luath Press, Edinburgh, 2014.

McEwen Nicola, Bomberg Elizabeth, "Sub-state Climate Pioneers: The Case of Scotland”, Regional \& Federal Studies 24(1), 2014, p. 63-85.

McInnes Colin, "Reclaim the inventive spirit of James Watt for an energy-rich, lower-carbon world", The Conversation, 2015.

PAquet Martin, «Contrat de communication et usages du passé : le cas de RadioCanada Atlantique ", dans Michel Beauchamp et Thierry Watine (dir.), Médias et milieux francophones, Les Presses de l'Université de Laval, Québec, 2006, p. 173-200.

Mechlin Hart, "It's Scotland's Oil: Energy and National Identity in Newspaper Coverage of Scottish Independence", The Journal of Politics and Society, vol. 26, $\mathrm{n}^{\circ} 1,2015$, p. 106-121.

Perron Dominique, Le nouveau roman de l'énergie nationale. Analyse des discours promotionnels d'Hydro-Québec de 1964 à 1997, University of Calgary Press, Calgary, 2006.

Piatti Marie-Christine, Les libertés individuelles à l'épreuve des nouvelles technologies de l'information, Presses universitaires de Lyon, Lyon, 2001.

Roche Sylvain, "Territorialiser la transition énergétique européenne : quand les îles écossaises veulent montrer l'exemple », Les Champs de Mars, Institut de recherche stratégique de l'École militaire (IRSEM), à paraître en 2019.

Rosenberg Dominique, Le principe de souveraineté des États sur leurs ressources naturelles, LGDJ, Paris, 1983.

Royal Society Of Edinburgh, Inquiry into Energy Issues for Scotland, Edinburgh, 2006.

SAVArD Stéphane, "Quand l'histoire donne sens aux représentations symboliques : L'Hydro-Québec, Manic-5 et la société québécoise ", Recherches sociographiques, vol. 50, $\mathrm{n}^{\circ} 1,2009$, p. 67-97.

Scottish Enterprise, Oil \& Gas Diversification Opportunities, Glasgow, 2017. 
Scottish Executive, Choosing Scotland's Future A National Conversation, Edinburgh, 2007.

sPaven David, Environmental politics in Scotland: towards the year 2000, The Scottish Government Yearbook, 1991.

Stevens Paul, "National oil companies and international oil companies in the Middle East: Under the shadow of government and the resource nationalism cycle", Journal of World Energy Law \& Business, n 1(1), 2008, p. 5-29.

The Scottish Government, A Low Carbon Economic Strategy for Scotland, Edinburgh, 2010.

The Scottish Government, 2020 Routemap for Renewable Energy in Scotland, Edinburgh, 2011.

The Scottish Government, Scotland's Future, Edinburgh, 2013a.

The Scottish Government, Scotland's Future: from the Referendum to Independence and a Written Constitution, Edinburgh, 2013b.

The Scottish Government, Scotland's Economy: the case for independence, Edinburgh, 2013c.

The Scottish Government, 2020 Routemap for renewable energy in Scotland, Edinburgh, 2013.

TAYLOR Alan, «Marine Renewables: A Distinctly Scottish Dimension », dans Wood G., Baker K. (éd), A Critical Review of Scottish Renewable and Low Carbon Energy Policy. Energy, Climate and the Environment, Palgrave Macmillan, Cham, 2017.

TETART Frank, "Les nationalismes "régionaux" en Europe, facteur de fragmentation spatiale ?", L'Espace politique, 11, 2010, URL : http://journals.openedition.org/espacepolitique/1647.

Therme Jean, "Les défis technologiques et industriels des énergies décarbonées ", Annales des Mines - Responsabilité et environnement, vol. 61, nº 1, 2011, p. 92-97.

Tном Françoise, "La naissance de l'énergocratie russe ", Commentaire, vol. 114, $n^{\circ} 2$, 2006, p. 295-300.

Toke David, Sherry-Brennan Fionnguala, Cowell Richard, Ellis Geraint Scotland, Strachan Peter, "Renewable energy and the independence debate: will head or heart rule the roost?", The Political Quarterly, 84(1), 2013, p. 61-70.

Toubal Louisa, L'investissement étranger, moteur de la réindustrialisation du Royaume-Uni ?, Transvalor/Presses des mines, Paris, 2018.

Tudi Kernalegenn, "Écologie et régionalisme ", Les notes de la Fondation de l'écologie politique, $\mathrm{n}^{\circ}$ 9, juin 2016, p. 1-16.

VEYRENC Thomas, "Un nouveau paradigme pour la politique énergétique européenne ?"Question d'Europe, $\mathrm{n}^{\circ}$ 163, Fondation Robert Schuman, 2010, URL : https://www.robert-schuman.eu/fr/questions-d-europe/0162-un-nouveau-paradigme-pour-la-politique-energetique-europeenne-1ere-partie. 
Sylvain Roche

\section{L'auteur}

Sylvain Roche est docteur en sciences économiques. Il a réalisé une thèse intitulée « Réenchanter le maritime par la promesse énergétique. Technologies, trajectoires, discours » soutenue en mai 2019 à l'Université de Bordeaux. Contact : sylvain.roche@u-bordeaux.fr 